\title{
Heavy quarkonia in a contact interaction and an algebraic model: mass spectrum, decay constants, charge radii and elastic and transition form factors
}

\author{
Khépani Raya, ${ }^{1}$ Marco A. Bedolla, ${ }^{1,2}$ J.J. Cobos-Martínez, ${ }^{3,4}$ and Adnan Bashir ${ }^{1}$ \\ 1 Instituto de Física y Matemáticas, Universidad Michoacana de San Nicolás de Hidalgo, \\ Edificio C-3, Ciudad Universitaria, Morelia, Michoacán 58040, México \\ ${ }^{2}$ Istituto Nazionale di Fisica Nucleare (INFN), Sezione di Genova, via Dodecaneso 33, 16146 Genova, Italy \\ 3 Laboratório de Física Teórica e Computacional, \\ Universidade Cruzeiro do Sul, 01506-000 São Paulo, Brasil \\ ${ }^{4}$ Cátedra CONACyT, Departamento de Física, Centro de Investigación \\ $y$ de Estudios Avanzados del Instituto Politécnico Nacional, \\ Apartado Postal 14-740, 07000, Ciudad de México, México
}

(Dated: November 2, 2017)

\begin{abstract}
For the flavor-singlet heavy quark system of bottomonia, we compute the masses of the ground state mesons in four different channels, namely, pseudo-scalar $\left(\eta_{b}(1 S)\right)$, vector $(\Upsilon(1 S))$, scalar $\left(\chi_{b_{0}}(1 P)\right)$ and axial vector $\left(\chi_{b_{1}}(1 P)\right)$. We also calculate the weak decay constants of the $\eta_{b}(1 S)$ and $\Upsilon(1 S)$ as well as the charge radius of $\eta_{b}(1 S)$. It complements our previous study of the corresponding charmonia systems: $\left.\eta_{c}(1 S), J / \Psi(1 S), \chi_{c_{0}}(1 P)\right)$ and $\left(\chi_{c_{1}}(1 P)\right)$. The unified formalism for this analysis is provided by a symmetry-preserving Schwinger-Dyson equations treatment of a vector $\times$ vector contact interaction. Whenever a comparison is possible, our results are in fairly good agreement with experimental data and model calculations based upon Schwinger-Dyson and BetheSalpeter equations involving sophisticated interaction kernels. Within the same framework, we also report the elastic and transition form factors to two photons for the pseudo-scalar channels $\eta_{c}(1 S)$ and $\eta_{b}(1 S)$ in addition to the elastic form factors for the vector mesons $J / \Psi$ and $\Upsilon$ for a wide range of photon momentum transfer squared $\left(Q^{2}\right)$. For $\eta_{c}(1 S)$ and $\eta_{b}(1 S)$, we also provide predictions of an algebraic model which correlates remarkably well between the known infrared and ultraviolet limits of these form factors.
\end{abstract}

PACS numbers: 12.38.-t, 11.10.St, 11.15.Tk, 14.40.Lb

\section{INTRODUCTION}

The discovery of heavy and narrow $J / \psi$ resonance in 1974 [1, 2] was followed by an even heavier and narrower $\Upsilon$ resonance in 1977 [3. These systems are characterized by two diametrically opposite energy scales: the hard scale of the heavy constituent quark masses and the soft scale of the relative momenta between them. They are bound systems yet their diminishing size with increasing mass probes coupling which tends to approach asymptotic freedom. Schwinger-Dyson equations (SDEs) of quantum chromodynamics (QCD) provide a natural means to study these systems; their derivation makes no assumption about the strength of the interaction involved. Therefore, they are ideally suited to study systems or phenomena which probe different energy scales simultaneously.

Earliest studies of heavy quarkonia through SDEs can be traced back to Ref. 4. With refined truncations of these equations and/or increased numerical complexity, these systems have also been investigated in Refs. [5] 14]. Predictions for states with exotic quantum numbers were made in Refs. [15, 16].

The extension of this program to the complicated exotic and baryonic states, decay rates and form factors is considerably non-trivial. Brute force numerical evaluation stops short of exploring the large momentum transfer region of form factors and, at times, is unable to make full comparison with already available experimental data. This shortcoming has been exposed in the calculation of elastic form factors (EFFs), see Ref. 17 for the pion EFF, as well as transition form factors (TFF), (see e.g., Ref. 18 for the two photon TFF of $\eta_{c}$ ). However, an artful parameterization of the Bethe-Salpeter amplitudes (BSAs) in terms of Nakanishi-like perturbation theory integral representations [19] allows us to reach large space-like momentum transfer region, see for example Refs. [14, 20, 21].

A few years ago, an alternative to full QCD based explorations was put forward to study pion properties assuming that quarks interact, not via massless vectorboson exchange, but instead through a symmetry preserving vector-vector contact interaction (CI) [22 26]. One then proceeds by embedding this interaction in a rainbow-ladder (RL) truncation of the SDEs. Confinement is implemented by employing a proper time regularization scheme. This scheme systematically removes quadratic and logarithmic divergences ensuring the axialvector Ward-Takahashi identity (axWTI) is satisfied. One can also explicitly verify the low energy GoldbergerTreiman relations. A fully consistent treatment of the CI model is simple to implement and can help us provide useful results which can be compared and contrasted with full QCD calculation and experiment.

This interaction is capable of providing a good description of the masses of meson and baryon ground and excited-states for light quarks 22 25]. The results de- 
rived from the CI model are quantitatively comparable to those obtained using sophisticated QCD model interactions [15, 27-29. Interestingly and importantly, this simple CI produces a parity-partner for each ground-state that is always more massive than its first radial excitation so that, in the nucleon channel, e.g., the first $J^{P}=1 / 2^{-}$ state lies above the second $J^{P}=1 / 2^{+}$state [24, 30].

Considering the discussion so far as a satisfactory justification, in Ref. [31, we extended this interaction model to the sector of heavy quarkonia, computing the mass spectrum of charmonia as well as the decay constants of the pseudoscalar and vector meson channels. A subsequent publication 32 applies this model to the computation of the EFF and the TFF of the $\eta_{c}$. Low momentum limit of the form factors allows us to extract charge radii which compare well with the earlier SDE computations and the lattice results. A symmetry-preserving CI has also been employed recently to study charmed mesons in [33.

Building on our efforts, we now extend this interaction to the analysis of the flavor-singlet heavy quark system of bottomonia, computing the masses of the ground state mesons in four different channels: pseudo-scalar $\left(\eta_{b}(1 S)\right)$, vector $(\Upsilon(1 S))$, scalar $\left(\chi_{b_{0}}(1 P)\right)$ and axial vector $\left(\chi_{b_{1}}(1 P)\right)$, as well as the weak decay constants of the $\eta_{b}(1 S)$ and $\Upsilon(1 S)$. We also compute the EFFs of $\eta_{b}(1 S), J / \psi$ and $\Upsilon(1 S)$, complementing our previous effort. Through evaluating the slope of these plots at the zero momentum transfer, we calculate and report their charge radii. We also calculate the transition form factor for $\eta_{b} \rightarrow \gamma^{*} \gamma$. Wherever possible, we compare our findings with experiment and/or other similar studies. Expectedly, the form factors are harder than we expect from the proper treatment of full QCD with a running quark mass function. As a quick fix, one resorts to the astutely constructed algebraic model (AM) and finds a near perfect substitute of cumbersome QCD based calculations.

The paper is organized as follows: in Section II we present the minimum but self contained details of the SDE-BSE approach to mesons; we also introduce the RL truncation for the $\mathrm{CI}$ and the consequences it has for the interaction kernels. We end the section by recalling the AM which mimics QCD to an adequate extent. In Section III. we tabulate our findings for the mass spectrum of the ground state bottomonia and decay constants of $\eta_{b}(1 S)$ and $\Upsilon(1 S)$. Section IV details algebraic expressions and numerical results for the EFFs of the pseudoscalar and vector mesons. It also contains a discussion on the quark-photon vertex we put to use, consistent with the WTI in the RL with a CI. Section $\nabla$ is devoted to the results and analysis of the transition form factors for the processes $\eta_{c(b)} \gamma^{*} \rightarrow \eta_{c(b)}$ and $\gamma \gamma^{*} \rightarrow \eta_{c(b)}$. Finally, in Section VI we summarize our conclusions.

\section{SDE-BSE FORMALISM}

We devote this section to the brief recapitulation of the SDE-BSE formalism to study two-particle bound states and their connection with chiral symmetry breaking. Our particular focuss will naturally be on the CI which is employed to produce the bulk of results reported in this article.

\section{A. The Gap Equation and the Contact Interaction}

The $f$-flavor dressed-quark propagator $S_{f}$ is obtained by solving the quark SDE [34 37.

$$
\begin{aligned}
& S_{f}^{-1}(p)=i \gamma \cdot p+m_{f}+\Sigma_{f}(p), \\
& \Sigma_{f}(p)=\int \frac{\mathrm{d}^{4} q}{(2 \pi)^{4}} g^{2} D_{\mu \nu}(p-q) \frac{\lambda^{a}}{2} \gamma_{\mu} S_{f}(q) \Gamma_{\nu}^{a}(p, q),
\end{aligned}
$$

where $g$ is the strong coupling constant, $D_{\mu \nu}$ is the dressed gluon propagator, $\Gamma_{\nu}^{a}$ is the dressed quark-gluon vertex, $m_{f}$ is the $f$-flavor current-quark mass and $\lambda^{a}$ are the usual Gell-Mann matrices.

Both $D_{\mu \nu}$ and $\Gamma_{\nu}^{a}$ satisfy their own SDEs, which in turn are coupled to higher $n$-point functions and so on ad infinitum. Therefore, the SDEs form an infinite set of coupled nonlinear integral equations, requiring a truncation scheme in order to define a tractable problem. This is achieved once we have specified the gluon propagator and the quark-gluon vertex. For a comprehensive recent review of the SDE-BSE formalism and its applications to hadron physics, see for example Refs. [27, 38.

The vector $\times$ vector $\mathrm{CI}$ assumes that the force mediation among quarks takes place not via massless vectorboson exchange but instead through the interaction defined by:

$$
\begin{aligned}
g^{2} D_{\mu \nu}(k) & =\frac{4 \pi \alpha_{\mathrm{IR}}}{m_{g}^{2}} \delta_{\mu \nu} \equiv \frac{1}{m_{G}^{2}} \delta_{\mu \nu}, \\
\Gamma_{\mu}^{a}(p, q) & =\frac{\lambda^{a}}{2} \gamma_{\mu},
\end{aligned}
$$

where $m_{g}=800 \mathrm{MeV}$ is a gluon mass scale generated dynamically in QCD (see for example Ref. [39]) and $\alpha_{\mathrm{IR}}$ is a parameter which specifies the enhanced interaction strength in the infrared (IR) [40, 41].

Equations (3) and (4) specify the kernel in the quark SDE, Eq. (1). In this truncation scheme, the general solution of the $f$-flavored dressed-quark propagator is immensely simplified 22, 26, 31, 32]:

$$
S_{f}^{-1}(p)=i \gamma \cdot p+M_{f} .
$$

As the interaction, Eqs. (34), is momentum independent, the fermion mass $M_{f}$ follows suit. This flavor-dependent constant mass is obtained by solving

$$
M_{f}=m_{f}+\frac{16 M_{f}}{3 \pi^{2} m_{G}^{2}} \int \frac{\mathrm{d}^{4} q}{(2 \pi)^{4}} \frac{1}{q^{2}+M_{f}^{2}} .
$$


Since the integral in Eq. (6) is divergent, we must adopt a regularization procedure. We employ the proper time regularization scheme 42 to write this equation as

$$
M_{f}=m_{f}+\frac{M_{f}^{3}}{3 \pi^{2} m_{G}^{2}} \Gamma\left(-1, \tau_{\mathrm{UV}}^{2} M_{f}^{2}, \tau_{\mathrm{IR}}^{2} M_{f}^{2}\right),
$$

where $\Gamma\left(a, z_{1}, z_{2}\right)$ is the generalized incomplete Gamma function:

$$
\Gamma\left(a, z_{1}, z_{2}\right)=\Gamma\left(a, z_{1}\right)-\Gamma\left(a, z_{2}\right) .
$$

The parameters $\tau_{\mathrm{IR}}$ and $\tau_{\mathrm{UV}}$ are, respectively, infrared and ultraviolet regulators. A nonzero value for $\tau_{\mathrm{IR}} \equiv$ $1 / \Lambda_{\mathrm{IR}}$ implements confinement by ensuring the absence of quark production thresholds 43 . Since the CI is not a renormalizable theory, $\tau_{\mathrm{UV}} \equiv 1 / \Lambda_{\mathrm{UV}}$ plays a dynamical role. Therefore, it sets the scale for all dimensioned quantities. The importance of an ultraviolet cutoff in Nambu-Jona-Lasinio type models has also been discussed in Refs. 44, 45.

Note that Eqs. (34) not only specify the kernel in the quark SDE, Eq. (1), but also the one in the BSE as we now make plain.

\section{B. The BSE and the Contact Interaction}

In quantum field theory, a meson bound state in a particular $J^{P C}$ channel is described by the BSE [46 48]

$$
\left[\Gamma_{H}(p ; P)\right]_{t u}=\int \frac{\mathrm{d}^{4} q}{(2 \pi)^{4}} K_{t u ; r s}(p, q ; P) \chi(q ; P)_{s r},
$$

where $\chi(q ; P)=S_{f}\left(q_{+}\right) \Gamma_{H}(q ; P) S_{g}\left(q_{-}\right) ; q_{+}=q+\eta P$, $q_{-}=q-(1-\eta) P ; \eta \in[0,1]$ is a momentum-sharing parameter, $p(P)$ is the relative (total) momentum of the quark-antiquark system; $S_{f(g)}$ is the $f(g)$-flavor dressedquark propagator, already discussed; $\Gamma_{H}(p ; P)$ is the meson Bethe-Salpeter amplitude (BSA), where $H$ specifies the quantum numbers and flavor content of the meson; $r, s, t$, and $u$ represent color, flavor, and spinor indices; and $K(p, q ; P)$ is the quark-antiquark scattering kernel.

Eqs. (34) define and relate the kernel of the gap equation with that of the BSE, Eq. (8), through the axialvector Ward-Takahashi identity (axWTI) 49]

$$
-i P_{\mu} \Gamma_{5 \mu}(k ; P)=S^{-1}\left(k_{+}\right) \gamma_{5}+\gamma_{5} S^{-1}\left(k_{-}\right) .
$$

Eq. (9), which encodes the phenomenological features of dynamical chiral symmetry breaking in QCD, relates the axial-vector vertex, $\Gamma_{5 \mu}(k ; P)$, to the quark propagator, $S(k)$. This in turn implies a relationship between the kernel in the BSE, Eq. (8), and that in the quark SDE, Eq. (1). This relation must be preserved by any viable truncation scheme of the SDE-BSE coupled system, thus constraining the content of the quark-antiquark scattering kernel $K(p, q ; P)$. For the CI, Eq. (9) implies

$$
K(p, q ; P)=-g^{2} D_{\mu \nu}(p-q)\left[\frac{\lambda^{a}}{2} \gamma_{\mu}\right] \otimes\left[\frac{\lambda^{a}}{2} \gamma_{\nu}\right],
$$

where $g^{2} D_{\mu \nu}$ is given by Eq. (3). Thus, the homogeneous $\operatorname{BSE}(\eta=1)$ takes the simple form

$\Gamma_{H}(p ; P)=-\frac{4}{3} \frac{1}{m_{G}^{2}} \int \frac{\mathrm{d}^{4} q}{(2 \pi)^{4}} \gamma_{\mu} S_{f}(q+P) \Gamma_{H}(q ; P) S_{g}(q) \gamma_{\mu}$.

The axWTI further implies [22, 26, 31, 32

$$
0=\int_{0}^{1} \mathrm{~d} x \int \frac{\mathrm{d}^{4} q}{(2 \pi)^{4}} \frac{\frac{1}{2} q^{2}+\mathfrak{M}^{2}}{\left(q^{2}+\mathfrak{M}^{2}\right)^{2}},
$$

where

$$
\mathfrak{M}^{2}=M_{f}^{2} x+M_{g}^{2}(1-x)+x(1-x) P^{2} .
$$

Eq. 12 has to be faithfully maintained during the entire calculation. It states that the axWTI is satisfied if, and only if, the model is regularized so as to ensure there are no quadratic or logarithmic divergences, circumstances under which a shift in integration variables is permitted. This is an essential requirement in order to prove Eq. (9) 22 25.

Since the interaction kernel of Eq. 10 does not depend on the external relative momentum, a symmetrypreserving regularization will return solutions which are independent of it. Therefore, the general forms of the BSAs for the pseudoscalar, scalar, vector, and axialvector channels read, respectively:

$$
\begin{aligned}
& \Gamma^{0^{-}}(P)=\gamma_{5}\left[i E^{0^{-}}(P)+\frac{1}{2 M} \gamma \cdot P F^{0^{-}}(P)\right] \\
& \Gamma^{0^{+}}(P)=\mathbb{1} E^{0^{+}}(P) \\
& \Gamma_{\mu}^{1^{-}}(P)=\gamma_{\mu}^{T} E^{1^{-}}(P)+\frac{1}{2 M} \sigma_{\mu \nu} P_{\nu} F^{1^{-}}(P) \\
& \Gamma_{\mu}^{1^{+}}(P)=\gamma_{5}\left[\gamma_{\mu}^{T} E^{1^{+}}(P)+\frac{1}{2 M} \sigma_{\mu \nu} P_{\nu} F^{1^{+}}(P)\right]
\end{aligned}
$$

where $M=M_{f} M_{g} /\left(M_{f}+M_{g}\right)$. The BSE is a homogeneous equation. Thus the BSA has to be normalized by a separate condition. In the RL approximation, this condition is:

$$
1=N_{c} \frac{\partial}{\partial P^{2}} \int \frac{\mathrm{d}^{4} q}{(2 \pi)^{4}} \operatorname{Tr}\left[\bar{\Gamma}_{H}(-Q) S(q+P) \Gamma_{H}(Q) S(q)\right]
$$

evaluated at $Q=P$, where $P^{2}=-m_{H}^{2}, \Gamma_{H}$ is the normalized BSA of the meson $H$, and $\bar{\Gamma}_{H}$ is its chargeconjugated version. For the vector and axial-vector channels, there is an additional factor of $1 / 3$ on the right-hand side to account for all three polarizations of a spin- 1 meson.

Once the BSA has been canonically-normalized, we can extract observables with it. The pseudoscalar and vector leptonic decay constants, $f_{0^{-}}$and $f_{1^{-}}$, are defined, respectively, by

$$
\begin{aligned}
P_{\mu} f_{0^{-}} & =N_{c} \int \frac{\mathrm{d}^{4} q}{(2 \pi)^{4}} \operatorname{Tr}\left[\gamma_{5} \gamma_{\mu} S\left(q_{+}\right) \Gamma_{0^{-}}(P) S\left(q_{-}\right)\right] \\
m_{V} f_{1^{-}} & =\frac{N_{c}}{3} \int \frac{\mathrm{d}^{4} q}{(2 \pi)^{4}} \operatorname{Tr}\left[\gamma_{\mu} S\left(q_{+}\right) \Gamma_{\mu}^{1^{-}} S\left(q_{-}\right)\right]
\end{aligned}
$$


where $m_{V}$ is the mass of the vector bound state, and the factor of 3 in the denominator corresponds to the three polarizations of the spin-1 meson.

\section{The Algebraic Model}

We present here a refined version of the previously employed AM for the heavy sector. For pseudoscalar mesons ( of mass $m_{P}$ ), it reads as:

$$
\begin{aligned}
\Gamma_{P}(k ; P) & =i \gamma_{5} \mathcal{N}_{P} \frac{M}{f_{P}} \int_{0}^{1} d z \rho_{\nu}(z) \frac{M^{2}}{k^{2}+z \sigma_{P} k \cdot P+M^{2}} \\
\rho_{\nu}(z) & =\frac{1}{\sqrt{\pi}} \frac{\Gamma[\nu+3 / 2]}{\Gamma[\nu+1]}\left(1-z^{2}\right)^{\nu}
\end{aligned}
$$

where $\mathcal{N}_{P}$ is the canonical normalization constant, $M$ is a mass scale (that we will relate to that of dynamical chiral symmetry breaking) and $f_{P}$ is the weak decay constant; $\sigma_{P} \equiv m_{\pi} / m_{P}$ is defined such that the angular dependence, $k \cdot P$, decreases as the meson mass increases, and it recovers the algebraic model for the pion when $m_{P}=m_{\pi}$. We pick $\nu=1$ in accordance with our earlier study of charmonia 32 .

Since there is no experimental value of $f_{\eta_{b}}$, we choose $M=4.818 \mathrm{GeV}$. It is a reasonable value since the dynamically generated constituent-like mass generated by the $\mathrm{CI}$ is $M=4.71 \mathrm{GeV}$. Moreover, it produces the $\eta_{b}$ weak decay constant which matches very well with the one reported in Ref. [13, based upon the most sophisticated SDE truncation so far. It is relevant to mention that this AM produces a finite-width delta-shaped parton distribution amplitude (PDA). Since this is the behavior of the PDA that one expects, one would also anticipate a decent prediction of the $\eta_{b}$ form factors.

We now turn to the computation of physical observables in the following sections.

\section{QUARKONIUM MASS SPECTRUM}

In Ref. [31, we extended the CI model to charmonia. Our findings for the ground-state mass spectrum of quark-model mesons are presented in TableI; see Ref. 31 for a discussion of these results. In this work, we carry out an identical exercise for bottomonia. Table II displays results for the ground-state mass spectrum in four different channels, obtained by solving Eq. (11). It can be readily inferred from Table II that our results are in excellent agreement with the experimental data and those computed more sophisticated/complex models. That a RL truncation with a CI describes the mass spectrum of ground-state heavy-quarkonia, charmonia and bottomonia, so well can be easily understood because the quark wave function renormalization is strictly one and the mass function is momentum independent. It implies that the heavy-quark-gluon vertex can reasonably be approximated by its bare counterpart [31, 32.

\begin{tabular}{c|c|c|c|c}
\hline \hline & \multicolumn{4}{|c}{ masses [GeV] } \\
\hline & $m_{\eta_{c}(1 S)}$ & $m_{J / \Psi(1 S)}$ & $m_{\chi_{c_{0}}(1 P)}$ & $m_{\chi_{c_{1}}(1 P)}$ \\
\hline Experiment [50] & 2.983 & 3.096 & 3.414 & 3.510 \\
CI [31] & 2.976 & 3.09 & 3.374 & 3.4 \\
JM [4] & 2.821 & 3.1 & 3.605 & - \\
BK [10] & 2.928 & 3.111 & 3.321 & 3.437 \\
RB [1] & 3.065 & - & - & - \\
FKW [1] & 2.925 & 3.113 & 3.323 & 3.489 \\
DGCLR [13] & 2.98 & 3.07 & - & - \\
\hline \hline
\end{tabular}

TABLE I: The ground-state charmonia mass spectrum. The tabulated CI results were obtained with the best-fit parameter set: $m_{g}=0.8 \mathrm{GeV}, \alpha_{I R}=0.93 \pi / 17=0.172, \Lambda_{\mathrm{IR}}=0.24 \mathrm{GeV}$ and $\Lambda_{\mathrm{UV}}=2.4 \mathrm{GeV}$. Note that the two parameters which differ from the light sector are $\alpha_{I R}$ and $\Lambda_{\mathrm{UV}}$ for the reasons explained within the text. The current charm quark mass is $m_{c}=1.09 \mathrm{GeV}$, whereas the dynamically generated constituent-like mass is $M_{c}=1.482 \mathrm{GeV}$. The average percentage error, with respect to experimental data, is less than $3 \%$. All subsequent calculation of charmonia related observables in this work employs the parameters listed this table.

\begin{tabular}{c|c|c|c|c}
\hline \hline & \multicolumn{4}{|c}{ masses [GeV] } \\
\hline & $m_{\eta_{b}(1 S)}$ & $m_{\Upsilon(1 S)}$ & $m_{\chi_{b_{0}}(1 P)}$ & $m_{\chi_{b_{1}}(1 P)}$ \\
\hline Experiment [50] & $9.32(9.4)$ & 9.46 & 9.860 & 9.892 \\
CI [This work] & 9.345 & 9.460 & 9.594 & 9.603 \\
JM [4] & 9.322 & 9.460 & 9.860 & - \\
BK [10] & 9.405 & 9.488 & 9.831 & 9.878 \\
FKW [12] & 9.414 & 9.490 & 9.815 & 9.842 \\
DGCLR [13] & 9.39 & 9.46 & - & - \\
MVRB [51] & - & 9.552 & - & - \\
\hline \hline
\end{tabular}

TABLE II: Ground-state bottomonia mass spectrum. The CI results were obtained with the modified parameters $\alpha_{I R}=$ $0.93 \pi / 125=0.023$ and $\Lambda_{\mathrm{UV}}=6.4 \mathrm{GeV}$. The current-quark mass is $m_{b}=3.8 \mathrm{GeV}$, and the dynamically generated constituentlike mass is $M_{b}=4.7 \mathrm{GeV}$. The average percentage error, with respect to experimental data, is again less than $3 \%$. All bottomonia related observables in this work have been evaluated by using the parameters listed this table.

The decay constants for the $\eta_{c}$ and $J / \Psi$ channels, computed in Ref. [31, are displayed in Table III, while the ones for the $\eta_{b}$ and $\Upsilon$ channels, calculated in the present work, are tabulated in Table IV. Our results are in reasonably good agreement for charmonia with about $20 \%$ deviation. There is no experimental value available for $f_{\eta_{b}}$. However, our result for $f_{\Upsilon}$ does not fare very favorably against the one obtained with the most sophisticated analysis of the SDEs to date [13, with the deviation stretching up to around $50 \%$.

As discussed at length in Ref. [31, we recall that the decay constant is influenced by the high momentum tail of the dressed-quark propagator and the BSAs [6, 57. 58, which probe the position space wave function of quarkonia at the origin. However, being momentumindependent, the CI results for the mass function and BSAs have no perturbative tail. This aspect is crucial 


\begin{tabular}{c|c|c}
\hline \hline & \multicolumn{2}{|c}{ decay constants [GeV] } \\
\hline & $f_{\eta_{c}}$ & $f_{J / \Psi}$ \\
Experiment & 0.238 & 0.294 \\
CI 31] & 0.255 & 0.206 \\
Lattice QCD & $0.279[52$ & $0.286[53]$ \\
BK [10] & 0.282 & 0.317 \\
KGH1 [54] & 0.284 & 0.318 \\
KGH2 [54] & 0.267 & 0.291 \\
DGCLR [13] & 0.262 & 0.255 \\
MVRB [51] & - & 0.306 \\
\hline
\end{tabular}

TABLE III: The decay constants for the states $\eta_{c}(1 S)$ and $J / \Psi(1 S)$. Note that the numerical values contain a division by $\sqrt{2}$ for a consistent comparison between different computations. The same is true for all subsequent discussion.

\begin{tabular}{c|c|c}
\hline \hline & \multicolumn{2}{|c}{ decay constants [GeV] } \\
\hline & $f_{\eta_{b}}$ & $f_{\Upsilon}$ \\
Experiment & - & 0.506 \\
CI [This work] & 0.553 & 0.219 \\
Lattice QCD & $0.472[55]$ & $0.459[56]$ \\
BK [10] & 0.501 & 0.486 \\
KGH1 [54] & 0.547 & 0.543 \\
KGH2 [54] & 0.535 & 0.500 \\
DGCLR [13] & 0.543 & 0.471 \\
\hline \hline
\end{tabular}

TABLE IV: The decay constants for the states $\eta_{b}(1 S)$ and $\Upsilon(1 S)$.

in a better prediction of the decay constants and would need to be built into the model in some indirect manner.

Moreover, as the quark masses become higher, mesons become increasingly pointlike in configuration space, thus weakening the interaction strength between their constituent quarks. It is this observation which led us to the natural extension of the CI model to heavy quarkonia by allowing a reduction of the effective coupling $\alpha_{I R}$, accompanied by an appropriate increase in the ultraviolet cutoff $\Lambda_{U V}$. However, we have retained the parameters $m_{g}$ and $\Lambda_{\mathrm{IR}}$ from the light sector. See Ref. 31], and references therein, for further discussion.

\section{A. On the Choice of Parameters}

\begin{tabular}{c|c|c|c|c}
\hline \hline quark & $\alpha_{I R}$ & $\Lambda_{U V}[\mathrm{GeV}]$ & $\alpha$ & Normalized \\
\hline$u, d, s$ & 2.922 & 0.905 & 3.739 & 1 \\
$c$ & 0.172 & 2.4 & 1.547 & 0.414 \\
$b$ & 0.023 & 6.4 & 1.496 & 0.400 \\
\hline \hline
\end{tabular}

TABLE V: The parameters of coupling $\alpha_{I R}$ and $\Lambda_{U V}$ are tabulated for different quark flavors. $m_{g}=0.8 \mathrm{GeV}$ and $\Lambda_{\mathrm{IR}}=0.24 \mathrm{GeV}$ are the fixed parameters. We also provide explicit mass-scale dependent $\alpha$ given by Eq. 21. The last column yields its normalized value such that $\alpha=1$ for the light quarks.
As mentioned previously, the only two parameters which are varied with the increasing quark masses are $\alpha_{I R}$ and $\Lambda_{U V}$. For the charmonia and bottomonia, these are determined from a best-fit to the mass and decay constant of the pseudoscalar channel. Those for the lightquarks sector were first obtained in Ref. [22, 24]. The arguments detailed earlier lead us to decrease $\alpha_{I R}$ with the increasing quark mass. This change has necessarily to be accompanied by a corresponding increase of $\Lambda_{U V}$ to ensure we get the observed mass and decay constant of the pseudoscalar meson. One may ask if there is a quantitative pattern in the variation of $\alpha_{I R}$ and $\Lambda_{U V}$. In that case, a choice of $\Lambda_{U V}$ will guide us towards the corresponding value of $\alpha_{I R}$.

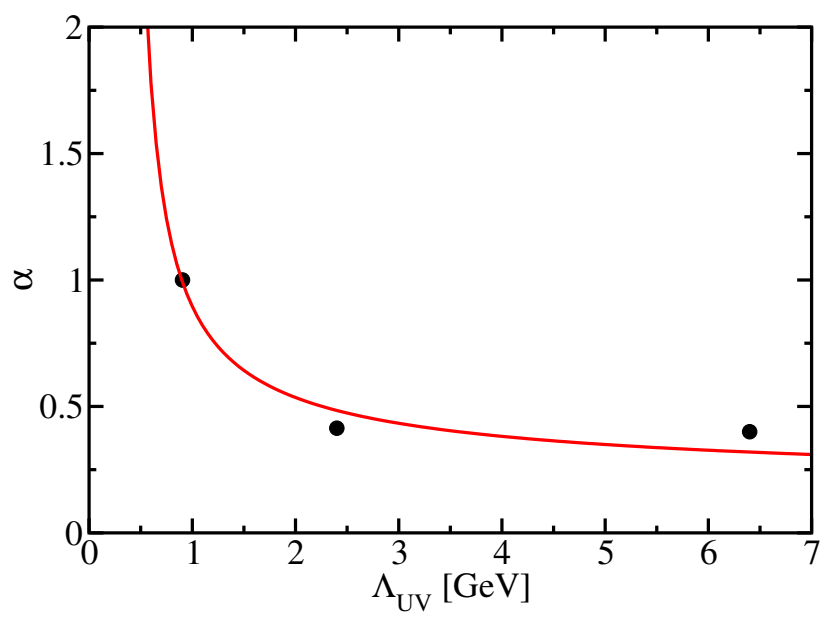

FIG. 1: Dimensionless coupling $\alpha$ for the contact interaction; see text. It is interesting to note that the variation of coupling $\alpha$ as a function of $\Lambda_{U V}$ is not far from a logarithmic decrease fitted by the function $\alpha=a \log ^{-1}\left(\Lambda_{U V} / \Lambda_{0}\right)$, where $\Lambda_{0}=$ $0.357 \mathrm{GeV}$ and $a=0.923$.

For this purpose, we define the following dimensionless coupling

$$
\alpha=\alpha\left(\Lambda_{U V}\right) \equiv \frac{\alpha_{I R}}{m_{g}^{2}} \Lambda_{U V}^{2}
$$

The advantage of this definition is that $\alpha$ naturally varies with the mass scale $\Lambda_{U V}$. This variation is plotted in Fig. 1. The decrease of $\alpha$, with respect to its value in the light-quarks sector, can be read off from the last column of Table $\mathrm{V}$. Indeed, $\alpha$ is reduced by a factor of $2.1-2.3$ in going from the light to the heavy sector (charmonia and bottomonia), instead of the apparent large factors quoted in Tables I and II. More importantly, we find out that the functional dependence of $\alpha\left(\Lambda_{U V}\right)$ can be fitted reasonably well with an inverse logarithmic curve, reminiscent of the running QCD coupling with the momentum scale at which it is measured. 


\section{ELASTIC FORM FACTORS}

Despite the fact that the charge-conjugation eigenstates, such as quarkonia, do not have an EFF, we can still couple a vector current to their constituent quarks. We expect this exercise to yield valuable information about the internal structure of the state [32, 59. Naturally, the vector current couples to the spin-1/2 quarks through the quark-photon interaction vertex. We briefly discuss this crucial ingredient in the following subsection.

\section{A. The Quark-photon Vertex}

It is of fundamental importance that quarks, whose dynamics inside hadrons is primarily dictated by QCD, also possess electromagnetic charge. The coupling of a photon with quarks plays an essential role in studying the internal structure of a hadron. Being an abelian theory, the only interaction of electromagnetic nature is the three-point quark-photon vertex.

The quark-photon vertex $\Gamma_{\mu}$ satisfies its own SDE and is constrained by the gauge invariance of QED through the vector Ward-Takahashi identity (WTI). Preserving this identity, and its $Q \rightarrow 0$ limit, is crucial to the conservation of electromagnetic current [17, 60. With our treatment of the CI, the bare vertex $\gamma_{\mu}$ is sufficient to satisfy the WTI and ensure a unit value for the charged pion's electromagnetic form factor at zero momentum transfer. However, given the simplicity of the model, one can readily improve upon it. A vertex dressed consistently with our truncation is determined by the following inhomogeneous BSE, (see Refs. 22, 26, 32, for more details):

$$
\Gamma_{\mu}(Q)=\gamma_{\mu}-\frac{4}{3 m_{G}^{2}} \int \frac{d^{4} q}{(2 \pi)^{4}} \gamma_{\alpha} \chi_{\mu}(q+P, q) \gamma_{\alpha},
$$

where $\chi_{\mu}(q+P, q)=S(q+P) \Gamma_{\mu}(Q) S(q)$. Therefore, the general form of the quark-photon vertex in the $\mathrm{RL}$ approximation with a CI is

$$
\Gamma_{\mu}(Q)=\gamma_{\mu}^{T} P_{T}\left(Q^{2}\right)+\gamma_{\mu}^{L} P_{L}\left(Q^{2}\right),
$$

where $Q_{\mu} \gamma_{\mu}^{T}=0$ and $\gamma_{\mu}^{T}+\gamma_{\mu}^{L}=\gamma_{\mu}$. The SDE for $\Gamma_{\mu}(Q)$, Eq. 22, implies

$$
\begin{aligned}
P_{L}\left(Q^{2}\right) & =1, \\
P_{T}\left(Q^{2}\right) & =\frac{1}{1-K_{V}\left(Q^{2}\right)},
\end{aligned}
$$

as can be consulted in Ref. 26, 32. $K_{V}\left(Q^{2}\right)$ is the BSE kernel in the vector channel, Eq. (15), within the present truncation scheme [31. Therefore, because of the dressing of the quark-photon vertex through $P_{T}\left(Q^{2}\right)$, our results for the form factors (EFF and TFF) will develop a pole at $Q^{2}=-m_{V}^{2}$, where $m_{1 V}$ is the vector meson mass. Note that $P_{T}\left(Q^{2}=0\right)=1$ and $P_{T}\left(Q^{2} \rightarrow \infty\right) \rightarrow 1$, leaving us with $\gamma_{\mu}$, as expected, the latter being the statement that a dressed-quark becomes a current quark to a large- $Q^{2}$ probe.

\section{B. $\eta_{c}$ and $\eta_{b}$ Elastic Form Factors}

A pseudoscalar meson possesses just one vector current form factor $F_{\eta_{c(b)}}\left(Q^{2}\right)$, defined by the $\eta_{c(b)} \gamma^{*}$ vertex

$$
\Lambda_{\mu}^{\eta_{c(b)} \gamma^{*}}\left(P_{i}, P_{f} ; Q\right)=F_{\eta_{c(b)}}\left(Q^{2}\right)\left(P_{i}+P_{f}\right)_{\mu},
$$

where $Q=P_{f}-P_{i}$ is the virtual photon momentum and $P_{i}\left(P_{f}\right)$ is that of the incoming (outgoing) meson. In our approach, the impulse approximation for the $\eta_{c(b)} \gamma^{*}$ vertex reads

$$
\begin{array}{r}
\Lambda_{\mu}^{\eta_{c(b)} \gamma^{*}}(P, Q)=2 N_{c} \int \frac{\mathrm{d}^{4} k}{(2 \pi)^{4}} \operatorname{Tr}\left[i \Gamma_{\eta_{c(b)}}\left(-P_{f}\right) S\left(k_{2}\right)\right. \\
\left.i \Gamma_{\mu}(Q) S\left(k_{1}\right) i \Gamma_{\eta_{c(b)}}\left(P_{i}\right) S(k)\right]
\end{array}
$$

where $\Gamma_{\mu}(Q)$ is the corresponding quark-photon vertex. Note that $P_{f}=P_{i}+Q$. We choose $P_{i}=P-Q / 2$. Thus $P_{f}=P+Q / 2, k_{1}=k+P-Q / 2$, and $k_{2}=k+P+Q / 2$. Since the scattering is elastic, $P_{i}^{2}=P_{f}^{2}=-m_{\eta_{c(b)}}^{2}$, which in turns implies $P \cdot Q=0$ and $P^{2}+Q^{2} / 4=-m_{\eta_{c(b)}}^{2}$, where $m_{\eta_{c(b)}}$ is the $\eta_{c(b)}$ mass.

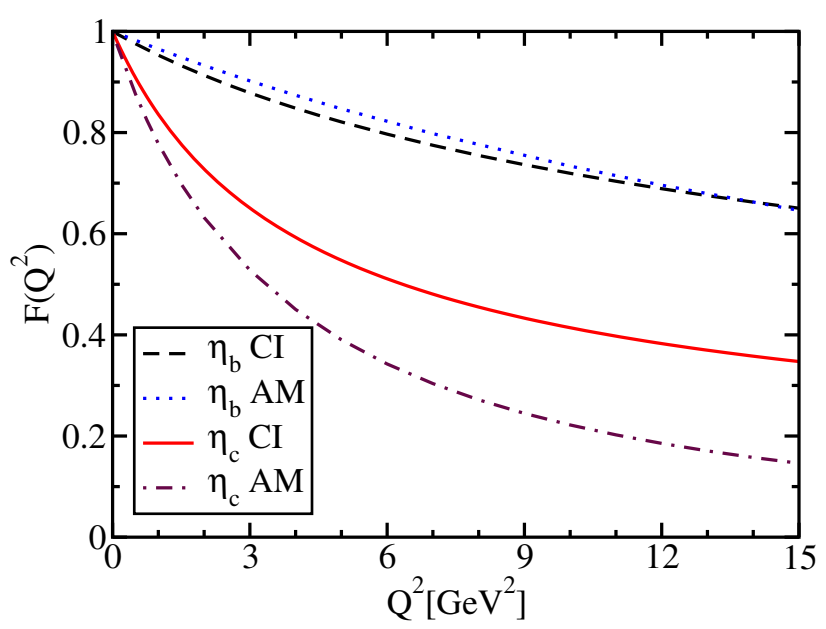

FIG. 2: We plot CI and AM results for the $\eta_{c}$ and $\eta_{b}$ EFFs. See Ref. 32 for a comparison between the CI result for the $\eta_{c} \mathrm{EFF}$ and that of lattice QCD from Ref. [59.

In Fig. 2, we display the $\eta_{c}$ and $\eta_{b}$ EFFs, calculated by using the dressed quark-photon vertex of Eq. (23). For heavy mesons, such as the $\eta_{c}$ and the $\eta_{b}$, the dressing of the vertex has a negligible effect on the EFF; that is, in the CI, the bare vertex trivially satisfies the WTI. However, although the time-like sector has not been displayed 
in Fig. 22 the $\eta_{c(b)}$ form factor has a pole at $Q^{2}=-m_{V}^{2}$, the mass of the corresponding vector bound state; see Tables II and II. This is a consequence of appropriately dressing the quark-photon vertex. For heavy quarks, the vector-meson pole lies deep on the time-like axis and hence hardly affects the form factors for the space-like $Q^{2}$. Wherever a comparison is possible, our results are harder than the ones predicted by the Lattice QCD [32]; see Ref. 32 for a detailed discussion.

Both for $\eta_{c}$ and $\eta_{b}$, the EFFs cease to depend on $Q^{2}$ for its sufficiently large values. This is the characteristic behavior of a point-like particle. In fact, it is already known that the asymptotic form factors obtained with the CI are harder than those predicted by QCD [22, 23, 26, 32]. This is an inevitable consequence of the momentum independence of the interaction, Eq. (3). As compared to $\eta_{b}$, the $\eta_{c}$ EFF increases more steeply for $Q^{2}<0$ since the pole (the $J / \Psi$ pole for the $\eta_{c}$ EFF) associated with the dressing of the $c$-quark-photon vertex lies closer to $Q^{2}=0$, naturally influencing the value of the charge radius defined as:

$$
r_{\eta_{c(b)}}^{2}=-\left.6 \frac{\mathrm{d} F_{\eta_{c(b)}}\left(Q^{2}\right)}{\mathrm{d} Q^{2}}\right|_{Q^{2}=0}
$$

Numerical values are presented in Table VI. The $\eta_{c}$ charge radius compares well with the prediction from Lattice QCD [59]. The charge radii of $\eta_{c}$ and $\eta_{b}$ are also in reasonably good agreement with SDE results with more sophisticated Maris-Tandy model interaction [7, 61. and with a light-front quantized Hamiltonian model [62]. Clearly $r_{\eta_{b}}<r_{\eta_{c}}$, i.e., the heavier the meson, the closer it is to being a point particle.

\begin{tabular}{c|c|c|c}
\hline \hline \multicolumn{4}{c}{ charge radius (fm) } \\
\hline & SDE & Lattice QCD & CI \\
\hline$\eta_{c}$ & $0.219[61]$ & $0.25[59,63]$ & $0.25[32]$ \\
$\eta_{b}$ & $0.086[7]$ & - & 0.109 [This work] \\
\hline \hline
\end{tabular}

TABLE VI: The charge radius for the state $\eta_{c(b)}(1 S)$ with the CI, compared to some other computations.

\section{C. $J / \Psi$ and $\Upsilon$ elastic form factors}

The electromagnetic structure of a spin-1 meson, like $J / \Psi$ and $\Upsilon$, is characterised by three vector current form factors [64]. We follow Ref. 61] in defining them.

$$
\Lambda_{\lambda \mu \nu}^{V \gamma^{*}}(P, Q)=\sum_{i=1}^{3} T_{\lambda \mu \nu}^{i}(P, Q) F_{i}\left(Q^{2}\right)
$$

where $V=J / \Psi, \Upsilon$ and $F_{i}, i=1,2,3$, are the EFFs. The kinematics remain identical to the case of pseudoscalar particles. However, the masses now refer to those of $M_{J / \Psi, \Upsilon}$. Defining the transverse projector

$$
\mathcal{P}_{\mu \nu}^{T}(P)=\delta_{\mu \nu}-\frac{P_{\mu} P_{\nu}}{P^{2}}
$$

the tensors in Eq. 29, are 61]:

$$
\begin{aligned}
T_{\lambda \mu \nu}^{1} & =2 P_{\lambda} \mathcal{P}_{\mu \alpha}^{T}\left(P^{i}\right) \mathcal{P}_{\alpha \nu}^{T}\left(P^{f}\right) \\
T_{\lambda \mu \nu}^{2} & =\left[Q_{\mu}-P_{\mu}^{i} \frac{Q^{2}}{2 m_{V}^{2}}\right] \mathcal{P}_{\lambda \nu}^{T}\left(P^{f}\right) \\
& -\left[Q_{\nu}+P_{\nu}^{f} \frac{Q^{2}}{2 m_{V}^{2}}\right] \mathcal{P}_{\lambda \mu}^{T}\left(P^{i}\right) \\
T_{\lambda \mu \nu}^{3} & =\frac{P_{\lambda}}{m_{V}^{2}}\left[Q_{\mu}-P_{\mu}^{i} \frac{Q^{2}}{2 m_{V}^{2}}\right]\left[Q_{\nu}+P_{\nu}^{f} \frac{Q^{2}}{2 m_{V}^{2}}\right]
\end{aligned}
$$

A symmetry-preserving regularization scheme is crucial so that the following WTIs are preserved [26]

$$
\begin{aligned}
Q_{\lambda} \Lambda_{\lambda \mu \nu}^{V \gamma^{*}} & =0, \\
P_{\mu}^{i} \Lambda_{\lambda \mu \nu}^{V \gamma^{*}} & =0=P_{\nu}^{f} \Lambda_{\lambda \mu \nu}^{V \gamma^{*}} .
\end{aligned}
$$

The first equation follows from current conservation and the latter two simply reflect the fact that the massive vector meson is transverse.

A more convenient and physically relevant set 64] of form factors, known as the charge, magnetic dipole, and electric quadrupole form factors is given by

$$
\begin{aligned}
G_{E}\left(Q^{2}\right) & =F_{1}\left(Q^{2}\right)+\frac{2}{3} \eta G_{Q}\left(Q^{2}\right) \\
G_{M}\left(Q^{2}\right) & =-F_{2}\left(Q^{2}\right) \\
G_{Q}\left(Q^{2}\right) & =F_{1}\left(Q^{2}\right)+F_{2}\left(Q^{2}\right)+(1+\eta) F_{3}\left(Q^{2}\right),
\end{aligned}
$$

where $\eta=Q^{2} /\left(4 m_{V}^{2}\right)$. In the limit $Q^{2} \rightarrow 0$, these form factors define the charge, magnetic dipole and electric quadrupole moments of the vector meson under consideration:

$$
\begin{aligned}
G_{E}\left(Q^{2}=0\right) & =1, \\
G_{M}\left(Q^{2}=0\right) & =\mu, \\
G_{Q}\left(Q^{2}=0\right) & =\mathcal{Q} .
\end{aligned}
$$

For a point-like vector particle, the magnetic and quadrupole moments are $\mu=2$ in units of $e /\left(2 m_{V}\right)$ and $\mathcal{Q}=-1$ in units of $e / m_{V}^{2}$, respectively [65].

In the impulse approximation the $V \gamma^{*}$ vertex can be calculated from the triangular configuration :

$$
\begin{array}{r}
\Lambda_{\lambda \mu \nu}^{V \gamma^{*}}(P, Q)=2 N_{c} \int \frac{\mathrm{d}^{4} k}{(2 \pi)^{4}} \operatorname{Tr}\left[\Gamma_{\nu}^{V}\left(-P^{f}\right) S\left(k+P^{f}\right)\right. \\
\left.i \Gamma_{\lambda}(Q) S\left(k+P^{i}\right) \Gamma_{\mu}^{V}\left(P^{i}\right) S(k)\right]
\end{array}
$$

Hence if one uses a symmetry-preserving regularisation, $G_{E}\left(Q^{2}=0\right)=1$ 26.

In Figures 3 and 4 , we plot our results for $G_{E}, G_{M}$, and $G_{Q}$ form factors for the vector mesons $J / \Psi$ and $\Upsilon$, 


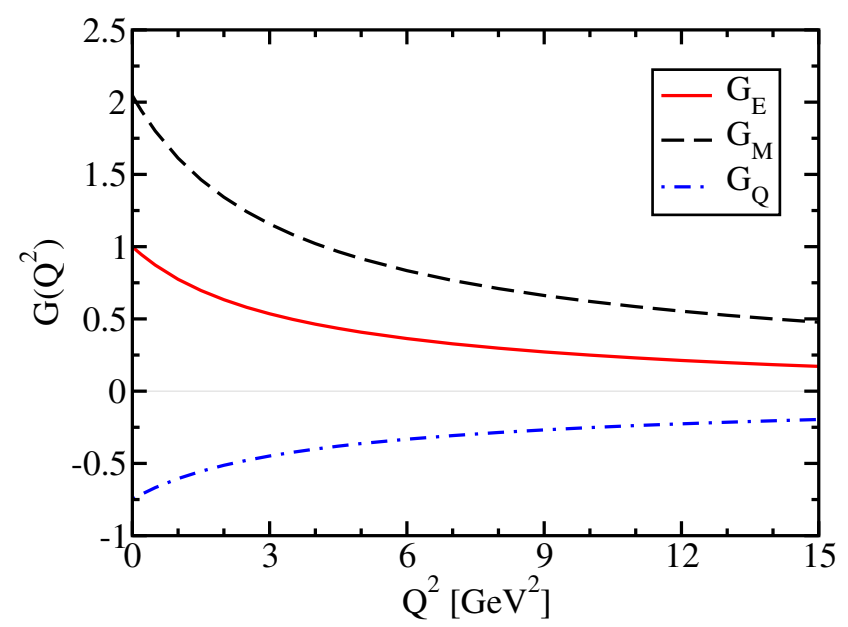

FIG. 3: Contact interaction results for the $J / \Psi$ EFFs $G_{E}$, $G_{M}$, and $G_{Q}$.

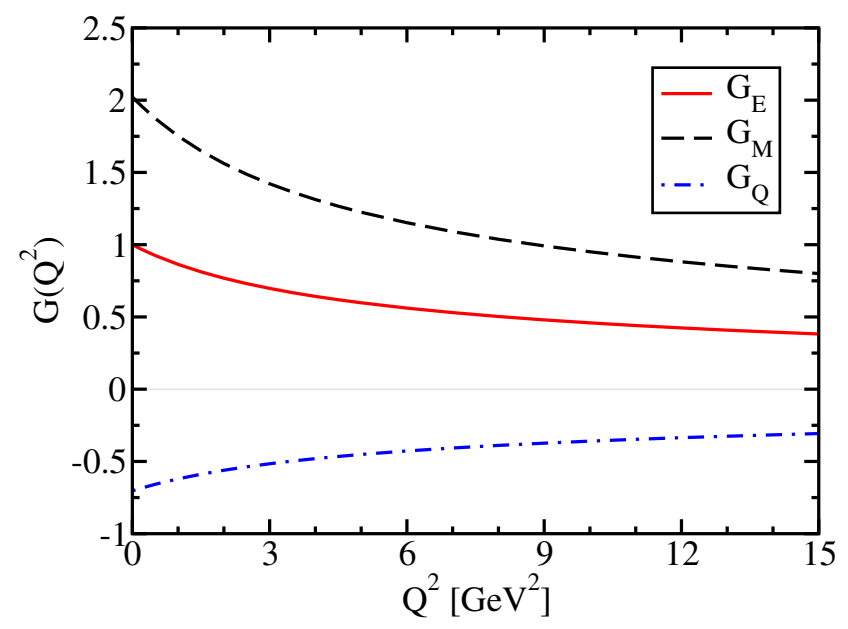

FIG. 4: Contact interaction plots for the $\Upsilon$ EFFs $G_{E}, G_{M}$, and $G_{Q}$.

respectively. As these form factors follow the same pattern as obtained for the $\rho$ meson form factors in Ref. [26], the same analysis applies here. We would like to mention that as compared to the pseudoscalars, these form factors fall faster, as $1 / Q^{2}$ for large $Q^{2}$ within the domain we explored, as opposed to the constant value observed for the pseudoscalars (see Fig. 2). This difference is due to the fact that the RL truncation with a CI prohibits the appearance of the vector component for the BSA of vector mesons, i.e., $F^{1^{-}}=0$ in Eq. 15. Asymptotic limit of QCD predicts 65]:

$$
\left.G_{E}\left(Q^{2}\right): G_{M}\left(Q^{2}\right): G_{(} Q^{2}\right) \stackrel{Q^{2}}{\rightarrow}=\infty-\frac{2}{3} \eta: 2:-1 .
$$

As noted in Ref. [26], this relation is recovered only for $\Lambda_{\mathrm{UV}} \rightarrow \infty$ but spoils the convergence of integrals, implying that a vector-vector CI cannot be regularized in a simple manner consistent with the constraints of asymptotic QCD.

\begin{tabular}{c|c|c|c|c|c}
\hline \hline & $r_{E}$ & $r_{M}$ & $r_{\mathcal{Q}}$ & $\mu$ & $\mathcal{Q}$ \\
\hline CI & 0.262 & 0.254 & 0.240 & 2.047 & -0.748 \\
SDE 61 & 0.228 & - & - & 2.13 & -0.28 \\
Lattice [59, 63 & 0.257 & - & - & 2.10 & -0.23 \\
\hline \hline
\end{tabular}

TABLE VII: Form factor radii (in fm) as well as magnetic and quadrupole moments for the $J / \Psi$ meson.

\begin{tabular}{c|c|c|c|c|c}
\hline \hline & $r_{E}$ & $r_{M}$ & $r_{\mathcal{Q}}$ & $\mu$ & $\mathcal{Q}$ \\
\hline CI & 0.197 & 0.195 & 0.182 & 2.012 & -0.704 \\
SDE & - & - & - & - & - \\
Lattice & - & - & - & - & - \\
\hline \hline
\end{tabular}

TABLE VIII: Form factor radii (in $\mathrm{fm}$ ) as well as magnetic and quadrupole moments for the $\Upsilon$ meson.

In Tables VII and VIII, we present charge, magnetization and quadrupole radii, as well as magnetic, and quadrupole moments for the $J / \Psi$ and $\Upsilon$, respectively. Reiterating the fact that for a structureless spin-1 particle, $\mu=2$ and $\mathcal{Q}=-1$ [65] any deviations from these values point to the dynamics of the internal structure. From the tabulated results, we can infer that the heavymesons produced by the CI are nearly point-like, as they should be. Wherever comparison is possible, our results of Table VII are in very good agreement with those of SDEs and Lattice.

\section{V. $\quad \gamma \gamma^{*} \rightarrow \eta_{c(b)}$ TRANSITION FORM FACTOR}

The interaction vertex describing the $\gamma \gamma^{*} \rightarrow \eta_{c(b)}$ transition can be parametrized by just one form factor $G_{\gamma \gamma^{*} \eta_{c(b)}}\left(Q_{1}^{2}, Q_{2}^{2}\right)$, which can be computed from

$$
\mathcal{T}_{\mu \nu}\left(Q_{1}, Q_{2}\right)=T_{\mu \nu}\left(Q_{1}, Q_{2}\right)+T_{\nu \mu}\left(Q_{2}, Q_{1}\right),
$$

where $Q_{1}$ and $Q_{2}$ are incoming photon momenta, $P=$ $Q_{1}+Q_{2}$ is the pseudoscalar's momentum, and the matrix element $T_{\mu \nu}\left(Q_{1}, Q_{2}\right)$ is given by

$$
\begin{aligned}
& T_{\mu \nu}\left(Q_{1}, Q_{2}\right)=\frac{\alpha_{\mathrm{em}}}{\pi f_{\eta_{c(b)}}} \epsilon_{\mu \nu \alpha \beta} Q_{1 \alpha} Q_{2 \beta} G_{\gamma \gamma^{*} \eta_{c(b)}}\left(Q_{1}^{2}, Q_{2}^{2}\right) \\
& =\operatorname{Tr} \int \frac{\mathrm{d}^{4} k}{(2 \pi)^{4}} S\left(k_{1}\right) \Gamma_{\eta_{c(b)}}(P) S\left(k_{2}\right) i \Gamma_{\mu}\left(Q_{2}\right) S\left(k_{3}\right) i \Gamma_{\nu}\left(Q_{1}\right),
\end{aligned}
$$

where $k_{1}=k-Q_{1}, k_{2}=k+Q_{2}, k_{3}=k$, and $\alpha_{\mathrm{em}}=$ $e^{2} /(4 \pi)$. In order to conform with the experimental set up, the kinematic constraints are $Q_{1}^{2}=Q^{2}$ and $Q_{2}^{2}=0$. 


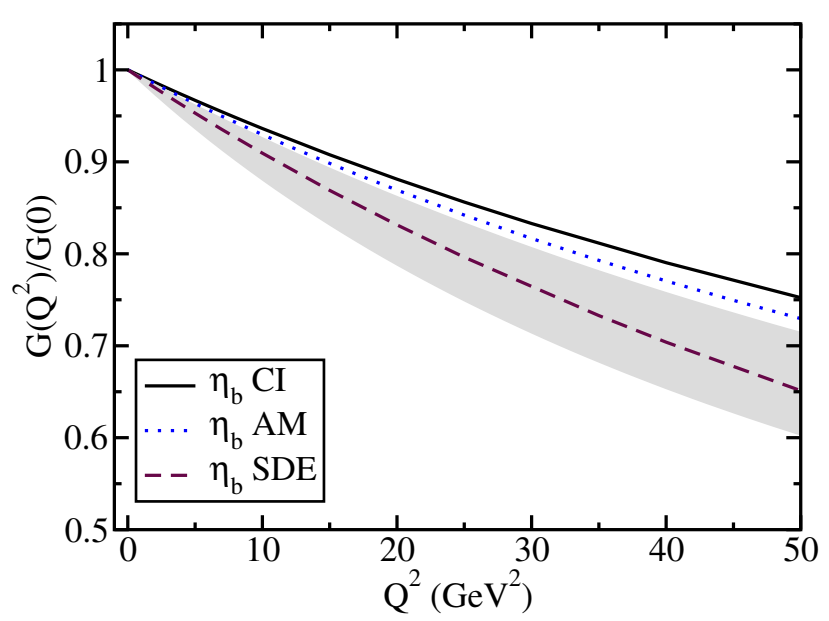

FIG. 5: CI results for the transition form factor for $\gamma \gamma^{*} \rightarrow \eta_{b}$ is represented by the (black) solid line. Blue (dotted) line stems from the the AM, Eq. 20). (Grey) band corresponds to the NNLO nrQCD result taken from Ref. 66. (the band width expresses the sensitivity to the factorization scale). (Purple) dashed line is the full QCD calculation of Ref. [14. As expected, AM does better than the $\mathrm{CI}$ in its connection with the QCD predictions, though the difference is not so conspicuous owing to the large mass of $\eta_{b}$.

We thus have $Q_{1} \cdot Q_{2}=-\left(m_{\eta_{c(b)}}^{2}+Q^{2}\right) / 2$, where $P^{2}=$ $-m_{\eta_{c(b)}}^{2}$.

\begin{tabular}{c|c|c|c|c}
\hline \hline \multicolumn{5}{c}{ interaction radius [fm] } \\
\hline & Experiment & Lattice QCD & SDE & CI \\
$\eta_{c}$ & 0.166 [67] & $0.141[59]$ & $0.16[14]$ & $0.133[32]$ \\
$\eta_{b}$ & - & - & $0.041[14]$ & 0.040 [This work] \\
\hline \hline
\end{tabular}

TABLE IX: Interaction radius of the transition $\gamma \gamma^{*} \rightarrow \eta_{c(b)}$ form factor as defined in Eq. 28. The experimental and lattice QCD results were extracted from their respective parametrization of data.

In Fig. 5, we present the CI results for the $\gamma \gamma^{*} \rightarrow$ $\eta_{c(b)}$ TFF. Although not shown in Fig. 5. both TFFs have a pole at $Q^{2}=-m_{V}^{2}$, as expected. For the $\eta_{c}$, the results compare fairly well with the $B A B A R$ data and Lattice QCD for low $Q^{2}$; see Ref. [32] for a more detailed analysis. However, for intermediate to large $Q^{2}$, the CI provides a harder form factor, and the correct asymptotic $Q^{2}$ behavior is not captured; see also Fig. 6 . We have neither experimental nor Lattice QCD data for the $\eta_{b} \mathrm{TFF}$ and interaction radius. However, we expect similar comparisons. Due to the same reason, in Fig. 5 . we could only provide the comparison of our findings with the NNLO nrQCD result [66] and a recent QCD based computation in the SDE-BSE formalism [14. Expectedly and encouragingly, our plots are in the same ballpark

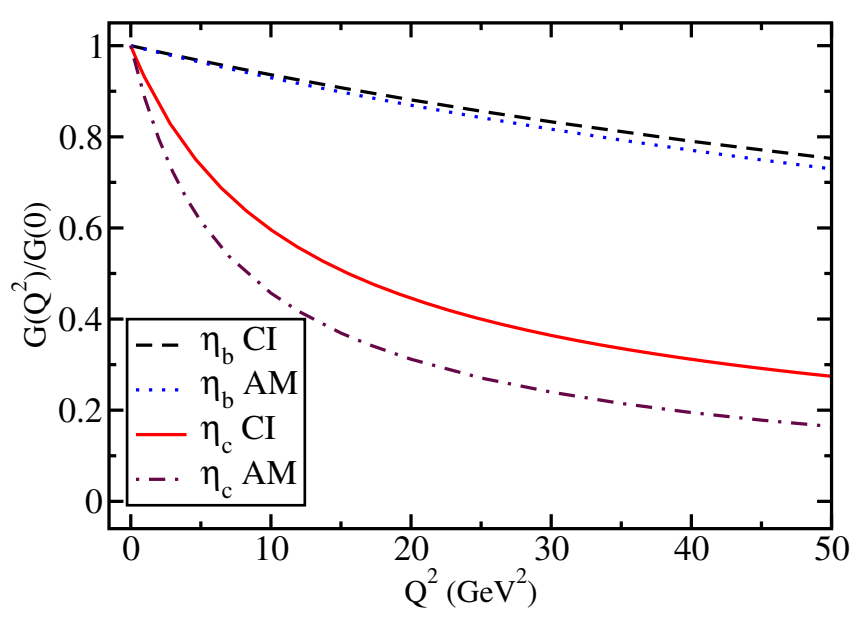

FIG. 6: Numerical results for $G\left(Q^{2}\right)$ for the transition $\gamma^{*} \gamma \rightarrow$ $\eta_{c, b}$, normalized to its value at $Q^{2}=0$. We compare the results for the CI and the AM. (Red) solid line is for the $\eta_{c}$ in the CI, compared to the (purple) dot-dashed line produced by the AM [32]. (Black) dashed curve is the CI prediction for the CI, whereas the (blue) dotted line is the AM result. The distinction between each pair of comparative curves reduces substantially as the meson gets heavier.

despite the simplicity of the model.

On the other hand, in Fig. 6, we present our results for $G_{\gamma \gamma^{*}} \eta_{c(b)}$. From this figure and Fig. 5 it can be deciphered that both TFFs tend to a constant value for large $Q^{2}$. However, the $\eta_{b}$ TFF reaches this limit faster. The interaction radius of the $\eta_{c}$ TFF, defined in Eq. (28) and tabulated in Table [X] compares well with Lattice QCD and the $B A B A R$ findings, as it probes the slope of the TFF for $Q^{2} \rightarrow 0$. It is also in good agreement with a recent, more sophisticated, SDE result [14. As with the $\eta_{c}$ 's interaction radius, we expect to achieve similar agreement for the $\gamma \gamma^{*} \rightarrow \eta_{b}$ transition. Indeed our, result for $r_{\eta_{b}}$ agrees nicely with that produced recently in Ref. [14. We hope to have corresponding Lattice QCD and experimental input at some point in future.

\section{CONCLUSIONS}

We compute the ground state spin-0 and spin-1 heavy quarkonia masses and decay constants by using a rainbow-ladder truncation of the simultaneous set of SDE and BSE within a CI model of QCD, developed initially for the light quarks sector 22,26$]$.

It was realized in Ref. 31, 32 that the extension of the CI model to charmonia requires a reduction of the interaction strength and a corresponding increase in the the ultraviolet cut-off. Present article is a generalization of this work to include bottomonia.

We find that the masses of the ground state heavy 
quarkonia are in good agreement with experimental results available [50] as well as SDE calculations with QCD based refined truncations [10, 13. The decay constants, however, are a bit lower. We have also computed the EFFs for $\eta_{c}, \eta_{b}, J / \Psi$, and $\Upsilon$, the transition form factors of the $\eta_{c}$ and $\eta_{b}$ and the corresponding radii.

The dressing of the heavy-quark-photon vertex, consistent with the model truncation and the WTI, ensures that the form factors posses a vector meson pole at $Q^{2}=-m_{V}^{2}$. However, since the vector meson mass is large, the effect of the meson vector pole on the charge radii is negligible. Our form factors, however, have better quantitative agreement with data and/or other calculations for small values of $Q^{2}$, whenever available. They are harder for intermediate and large $Q^{2}$. For the pseudoscalars, the both form factors tend to a constant for $Q^{2} \rightarrow \infty$, which is a consequence of the momentumindependent interaction. On the other hand, the vector component of the vector meson is zero and the corresponding EFFs tend to behave as $1 / Q^{2}$ for large $Q^{2}$.

Our results for charge radii are in very good agreement with Lattice QCD data and experimental results, whenever available; furthermore comparison between the charga radii for charmonia and bottomonia confirms that the heavier the meson the closer it is to being a point particle, justifying our reduction of the strength of the coupling.

All this is an encouraging first step towards a comprehensive study of heavy hadrons in this approach. Immediate next steps will involve flavored mesons and baryons. Our goal is to provide a unified description of light and heavy hadrons within the CI model.

\section{ACKNOWLEDGMENTS}

The authors acknowledge financial support from CONACyT, México (doctoral scholarship for M.A. Bedolla; postdoctoral Contract No. 290917UMSNH for J.J. Cobos-Martínez and research Grant No. CB-2014-242117 for A. Bashir). This work has also partly been financed by the CIC-UMSNH Grant 4.10. J.J. Cobos-Martínez also acknowledges the support of Conselho Nacional de Desenvolvimento Cientifico e Tecnologico (CNPq,Brazil), Grant No. 152348/2016-6 and Instituto Nacional de Ciencia e Tecnologia - Fisica Nuclear e Aplicações (INCT-FNA, Brazil) CAPES: 88887.145710/2017-00.
[1] J. J. Aubert et al. (E598), Phys. Rev. Lett. 33, 1404 (1974).

[2] J. E. Augustin et al. (SLAC-SP-017), Phys. Rev. Lett. 33, 1406 (1974), [Adv. Exp. Phys.5,141(1976)].

[3] S. W. Herb et al., Phys. Rev. Lett. 39, 252 (1977).

[4] P. Jain and H. J. Munczek, Phys.Rev. D48, 5403 (1993), hep-ph/9307221.

[5] A. Krassnigg and P. Maris, J.Phys.Conf.Ser. 9, 153 (2005), nucl-th/0412058.

[6] M. Bhagwat, A. Holl, A. Krassnigg, C. Roberts, and P. Tandy, Phys.Rev. C70, 035205 (2004), nuclth/0403012.

[7] M. Bhagwat, A. Krassnigg, P. Maris, and C. Roberts, Eur.Phys.J. A31, 630 (2007), nucl-th/0612027.

[8] P. Maris and P. Tandy, Nucl.Phys.Proc.Suppl. 161, 136 (2006), nucl-th/0511017.

[9] N. Souchlas, Phys.Rev. D81, 114019 (2010).

[10] M. Blank and A. Krassnigg, Phys.Rev. D84, 096014 (2011), 1109.6509.

[11] E. Rojas, B. El-Bennich, and J. de Melo, Phys.Rev. D90, 074025 (2014), 1407.3598.

[12] C. S. Fischer, S. Kubrak, and R. Williams, Eur. Phys. J. A51, 10 (2015), 1409.5076.

[13] M. Ding, F. Gao, L. Chang, Y.-X. Liu, and C. D. Roberts, Phys. Lett. B753, 330 (2016), 1511.04943.

[14] K. Raya, M. Ding, A. Bashir, L. Chang, and C. D. Roberts, Phys. Rev. D95, 074014 (2017), 1610.06575.

[15] P. Maris, AIP Conf.Proc. 892, 65 (2007), nuclth/0611057.

[16] A. Krassnigg, Phys.Rev. D80, 114010 (2009), 0909.4016.

[17] P. Maris and P. C. Tandy, Phys. Rev. C62, 055204 (2000), nucl-th/0005015.
[18] J. Chen, M. Ding, L. Chang, and Y.-x. Liu, Phys. Rev. D95, 016010 (2017), 1611.05960.

[19] N. Nakanishi, Phys. Rev. 130, 1230 (1963).

[20] L. Chang, I. C. Clöt, C. D. Roberts, S. M. Schmidt, and P. C. Tandy, Phys. Rev. Lett. 111, 141802 (2013), 1307.0026.

[21] K. Raya, L. Chang, A. Bashir, J. J. Cobos-Martinez, L. X. Gutiérrez-Guerrero, C. D. Roberts, and P. C. Tandy, Phys. Rev. D93, 074017 (2016), 1510.02799.

[22] L. Gutierrez-Guerrero, A. Bashir, I. Cloet, and C. Roberts, Phys.Rev. C81, 065202 (2010), 1002.1968.

[23] H. Roberts, C. Roberts, A. Bashir, L. GutierrezGuerrero, and P. Tandy, Phys.Rev. C82, 065202 (2010), 1009.0067.

[24] C. Chen, L. Chang, C. D. Roberts, S. Wan, and D. J. Wilson, Few Body Syst. 53, 293 (2012), 1204.2553.

[25] H. L. Roberts, L. Chang, I. C. Cloet, and C. D. Roberts, Few Body Syst. 51, 1 (2011), 1101.4244.

[26] H. Roberts, A. Bashir, L. Gutierrez-Guerrero, C. Roberts, and D. Wilson, Phys.Rev. C83, 065206 (2011), 1102.4376.

[27] A. Bashir, L. Chang, I. C. Cloet, B. El-Bennich, Y.X. Liu, et al., Commun.Theor.Phys. 58, 79 (2012), 1201.3366.

[28] G. Eichmann, R. Alkofer, I. Cloet, A. Krassnigg, and C. Roberts, Phys.Rev. C77, 042202 (2008), 0802.1948.

[29] I. Cloet, A. Krassnigg, and C. Roberts, eConf C070910, 125 (2007), 0710.5746.

[30] Y. Lu, C. Chen, C. D. Roberts, J. Segovia, S.-S. Xu, and H.-S. Zong, Phys. Rev. C96, 015208 (2017), 1705.03988.

[31] M. A. Bedolla, J. Cobos-Martinez, and A. Bashir, Phys. Rev. D92, 054031 (2015). 
[32] M. A. Bedolla, K. Raya, J. Cobos-Martnez, and A. Bashir, Phys. Rev. D93, 094025 (2016).

[33] F. E. Serna, B. El-Bennich, and G. Krein, Phys. Rev. D96, 014013 (2017), 1703.09181.

[34] C. D. Roberts, M. S. Bhagwat, A. Holl, and S. V. Wright, Eur. Phys. J. ST 140, 53 (2007), 0802.0217.

[35] A. Holl, C. D. Roberts, and S. V. Wright, in 20th Annual Hampton University Graduate Studies Program (HUGS 2005) Newport News, Virginia, May 31-June 17, 2005 (2006), nucl-th/0601071.

[36] P. Maris and C. D. Roberts, Int. J. Mod. Phys. E12, 297 (2003), nucl-th/0301049.

[37] R. Alkofer and L. von Smekal, Phys. Rept. 353, 281 (2001), hep-ph/0007355.

[38] I. G. Aznauryan et al., Int. J. Mod. Phys. E22, 1330015 (2013), 1212.4891.

[39] P. Boucaud, J. Leroy, A. L. Yaouanc, J. Micheli, O. Pene, et al., Few Body Syst. 53, 387 (2012), 1109.1936.

[40] D. Binosi, C. Mezrag, J. Papavassiliou, C. D. Roberts, and J. Rodriguez-Quintero (2016), 1612.04835.

[41] A. Deur, S. J. Brodsky, and G. F. de Teramond, Prog. Part. Nucl. Phys. 90, 1 (2016), 1604.08082.

[42] D. Ebert, T. Feldmann, and H. Reinhardt, Phys.Lett. B388, 154 (1996), hep-ph/9608223.

[43] C. Roberts, Prog.Part.Nucl.Phys. 61, 50 (2008), 0712.0633 .

[44] R. Farias, G. Dallabona, G. Krein, and O. Battistel, Phys.Rev. C73, 018201 (2006), hep-ph/0510145.

[45] R. Farias, G. Dallabona, G. Krein, and O. Battistel, Phys.Rev. C77, 065201 (2008), hep-ph/0604203.

[46] F. Gross, Relativistic quantum mechanics and field theory (Wiley, New York, 1993), 1st ed.

[47] E. E. Salpeter and H. A. Bethe, Phys. Rev. 84, 1232 (1951).

[48] M. Gell-Mann and F. Low, Phys. Rev. 84, 350 (1951).

[49] P. Maris, C. D. Roberts, and P. C. Tandy, Phys. Lett. B420, 267 (1998), nucl-th/9707003.

[50] K. Nakamura and P. D. Group, Journal of Physics G: Nuclear and Particle Physics 37, 075021 (2010), URL http:
//stacks.iop.org/0954-3899/37/i=7A/a=075021.

[51] F. F. Mojica, C. E. Vera, E. Rojas, and B. El-Bennich, Phys. Rev. D96, 014012 (2017), 1704.08593.

[52] C. T. H. Davies, C. McNeile, E. Follana, G. P. Lepage, H. Na, and J. Shigemitsu, Phys. Rev. D82, 114504 (2010), 1008.4018.

[53] G. C. Donald, C. T. H. Davies, R. J. Dowdall, E. Follana, K. Hornbostel, J. Koponen, G. P. Lepage, and C. McNeile, Phys. Rev. D86, 094501 (2012), 1208.2855.

[54] A. Krassnigg, M. Gomez-Rocha, and T. Hilger, J. Phys. Conf. Ser. 742, 012032 (2016), 1603.07232.

[55] C. McNeile, C. T. H. Davies, E. Follana, K. Hornbostel, and G. P. Lepage, Phys. Rev. D86, 074503 (2012), 1207.0994.

[56] B. Colquhoun, R. J. Dowdall, C. T. H. Davies, K. Hornbostel, and G. P. Lepage, Phys. Rev. D91, 074514 (2015), 1408.5768.

[57] P. Maris and C. D. Roberts, Phys. Rev. C56, 3369 (1997), nucl-th/9708029.

[58] P. Maris and P. C. Tandy, Phys. Rev. C60, 055214 (1999), nucl-th/9905056.

[59] J. J. Dudek, R. G. Edwards, N. Mathur, and D. G. Richards, J.Phys.Conf.Ser. 69, 012006 (2007).

[60] P. Maris and P. C. Tandy, Phys. Rev. C61, 045202 (2000), nucl-th/9910033.

[61] M. S. Bhagwat and P. Maris, Phys. Rev. C77, 025203 (2008), nucl-th/0612069.

[62] Y. Li, P. Maris, and J. P. Vary, Phys. Rev. D96, 016022 (2017), 1704.06968.

[63] J. J. Dudek, R. G. Edwards, and D. G. Richards, Phys. Rev. D73, 074507 (2006), hep-ph/0601137.

[64] R. G. Arnold, C. E. Carlson, and F. Gross, Phys. Rev. C21, 1426 (1980).

[65] S. J. Brodsky and J. R. Hiller, Phys. Rev. D46, 2141 (1992).

[66] F. Feng, Y. Jia, and W.-L. Sang, Phys. Rev. Lett. 115, 222001 (2015), 1505.02665.

[67] V. P. Druzhinin (BaBar), PoS ICHEP2010, 144 (2010), 1011.6159. 